\section{Cytoplasmic lattices are not linked to mouse 2-cell embryos developmental arrest}

\author{
Marianna Longo, ${ }^{1 \#}$ Michele Boiani, ${ }^{2}$ \\ Carlo Alberto Redi, ${ }^{3}$ Manuela Monti ${ }^{1}$ \\ ${ }^{1}$ Research Center for Regenerative \\ Medicine, Biotechnologies Research \\ Laboratories, Fondazione IRCCS \\ Policlinico San Matteo, Pavia, Italy \\ ${ }^{2}$ Max Planck Institute for Molecular \\ Biomedicine, Muenster, Germany \\ ${ }^{3}$ Department of Biology and \\ Biotechnology "L. Spallanzani", \\ University of Pavia, Italy \\ \#Present address: MRC Protein \\ Phosphorylation and Ubiquitylation \\ Unit, School of Life Sciences, University \\ of Dundee, UK
}

\begin{abstract}
Cytoplasmic lattices are important regulators of oocyte maturation. They store components of the protein synthesis machinery including ribosomes and, among others, they are involved in the regulation of microtubule dynamics in both mouse and human. Cytoplasmic lattices undergo dramatic reorganizations at crucial stages of oocyte maturation, where they are abundantly present in the cytoplasm of developmentally competent oocytes named SN (Surrounded Nucleolus) while they are rare in the cytoplasm of 2-cell stage-arresting NSN (Not Surrounded Nucleolus) oocytes, suggestive of a requirement of cytoplasmic lattices for development past the 2-cell stage. Here, to elucidate this requirement, 2cell mouse embryos derived from $\mathrm{SN}$ and NSN oocytes were analyzed by transmission electron microscopy. Contrary to what had been proposed hitherto, cytoplasmic lattices are present in 2-cell embryos derived not only from $\mathrm{SN}$, but also from NSN oocytes, irrespective of the embryo production system (intracytoplasmic sperm injection, parthenogenesis). Hence our conclusion that cytoplasmic lattices do not count among the factor(s) responsible for the embryo arrest at this crucial stage of development.
\end{abstract}

\section{Introduction}

The acquisition of oocyte developmental competence is one of the most essential and fascinating biological processes, con- sisting of several steps leading to meiosis progression and to the ability to sustain fertilization and the prospect embryo development.

Several factors and mechanisms are involved in each phase of this process like the control of oocyte development by the interaction with somatic cells, the arrangement of the chromosomes within the nucleus, the organelles rearrangement, the role played by epigenetic mechanisms, the chromatin conformation of the NSN oocytes and the down regulation of maternal genes/proteins are the most reasonable explanations of the embryo arrest at the 2/4-cell stage. ${ }^{1-4}$

Among the organelles of the ooplasm, cytoplasmic lattices (CPLs) are ascribed with key roles in oocyte maturation, although their degree of involvement is not entirely understood. CPLs are causatively related to microtubule and organelle dynamics, ribosomal storage and maternalzygotic transition by controlling the translational processes, ${ }^{3,5}$ they are also (apparently) required for the embryo development beyond the 2-cell stage: ${ }^{6}$ in other words, CPLs are functionally relevant to the housekeeper (basic) as well as luxury biological processes. Recent evidence showed that CPLs start to appear in primary oocytes and function as a storage site for aggregated maternal ribosomes. ${ }^{6}$ CPLs have also been found abundant in the cytoplasm of mouse and human germinal vesicle (GV) oocytes named Surrounded Nucleolus (SN), which account for $70-80 \%$ of GV oocytes that are able to develop beyond the 2/4-cell stage after maturation and fertilization; conversely, the remaining $20-30 \%$ of oocytes named Not Surrounded Nucleolus (NSN) are almost completely devoid of CPLs and also lack the ability to develop beyond the 2/4cell stage. ${ }^{7,8}$ Based on these records, we hypothesized that presence/absence of CPLs in SN/NSN GV oocytes is linked to the occurrence of 2-cell stage arrest, as also suggested by recent studies on maternal proteins essential for embryo development beyond the 2/4-cell stage. ${ }^{4}$ Indeed, MATER, a main constituent of the subcortical maternal complex (SCMC) plays critical roles in CPLs formation and function and in embryo development beyond the 2-cell stage. ${ }^{4}$ It has been shown that the majority of GV oocytes isolated from animals with a transient mutation for MATER have an NSN phenotype with no CPLs in their cytoplasm ${ }^{7}$ and, as expected, are unable to support the embryonic development beyond the 2-cell stage. Interestingly, the depletion of other maternal proteins and/or members of the SCMC, like PADI6, probably leads to the formation of NSN GV oocytes (and/or prevents the transition from NSN to SN) with no CPLs
Correspondence: Dr. Manuela Monti, Research Center for Regenerative Medicine, Biotechnologies Research Laboratories, Fondazione IRCCS Policlinico San Matteo, viale Golgi 19, 27100 Pavia, Italy.

E-mail:m.monti@smatteo.pv.it

Keywords: Oocytes; cytoplasmic lattices; transmission electron microscopy; 2-cell embryo; developmental competence; ICSI; parthenogenesis.

Contributions: ML, MB, collected the oocytes and performed in vitro maturation, parthenogenetic and ICSI experiments; MM, CAR, conceived the study, analyzed the images and performed the quantitative analysis; MM, CAR wrote the manuscript; MB, participated in manuscript revision. All authors read and approved the final manuscript.

Funding: This work was supported by grant $n$ 688-rcr2012-50 from Fondazione IRCCS Policlinico San Matteo to MM.

Acknowledgments: The authors wish to thank Alberto Calligaro for discussion and Dagmar Zeuschner, Karina Mildner, Ellen Casser and Steffen Israel for technical help with transmission electron microscopy samples preparation.

Conflict of interest: The authors declare they have no competing interests.

Received for publication: 30 July 2018.

Accepted for publication: 14 September 2018.

This work is licensed under a Creative Commons Attribution-NonCommercial 4.0 International License (CC BY-NC 4.0).

(C) Copyright M. Longo et al., 2018

Licensee PAGEPress, Italy

European Journal of Histochemistry 2018; 62:2972 doi:10.4081/ejh.2018.2972

and unable to complete the prospect embryo development. ${ }^{6,9,10}$ Based on these records, we sought to elucidate the requirement of CPLs for preimplantation development, by quantitatively analyzing the CPLs content in 2-cell embryos of SN and NSN oocytes; specifically, we tested the expectation that only SN-derived embryos have CPLs in their cytoplasm. Thanks to the use of two embryo production systems, namely parthenogenesis and fertilization (intracytoplasmic sperm injection, ICSI), we were able to exclude a differential response of SN and NSN oocytes to sperm, and to retain the pristine nature of the oocytes in respect to the cytoplasmic lattices, which are of maternal origin. The evidence here provided supports the view that cytoplasmic lattices are not linked to mouse 2-cell embryos developmental arrest. 


\section{Materials and Methods}

\section{Ethics statement}

All mice (20 females and 4 males) used were housed in individually ventilated cages in the animal facility of the MPI Münster, with a controlled temperature of $22^{\circ} \mathrm{C}$, with a $14 / 10 \mathrm{~h} \mathrm{light/dark} \mathrm{photoperiod}$ and with free access to water and food (Harlan Teklad 2020SX). Experimental procedures followed the ethical guidelines of the European Laboratory Animal Science Associations (FELASA) and the regulatory guidelines of European Directive 63/2010. On the local regulatory level, mice were used for experiments according to the ethical permit issued by the Landesamt für Natur, Umwelt und Verbraucherschutz (LANUV) of the state of North RhineWestphalia, Germany (permit number 8402.05.20.13.022).

\section{Collection of oocytes and spermatozoa}

Six to eight week-old B6C3F1 female mice were used as donors of GV-stage oocytes for in vitro maturation (IVM) and metaphase II (MII)-stage oocytes for developmental activation (intracytoplasmic sperm injection, ICSI; parthenogenesis). Twelve week-old CD1 male mice were used as sperm donors for ICSI. GV-oocytes were isolated by puncturing the surface of the ovary $48 \mathrm{~h}$ after PMSG stimulation (10 IU), MII oocytes were recovered from the oviductal ampullae $15 \mathrm{~h}$ after hCG and PMSG stimulation injected $48 \mathrm{~h}$ apart (10 IU each), and freed of cumulus cells using hyaluronidase $(50 \mathrm{IU} / \mathrm{mL})$. Caudae epididymis were punctured with a $27 \mathrm{G}$ needle, and overlaid with pre-equilibrated Whittingham medium ( $3 \%$ BSA) on the bottom of a $1.5 \mathrm{~mL}$ Eppendorf tube so as to allow for sperm to swim-up at $37^{\circ} \mathrm{C}$ under $5 \% \mathrm{CO}_{2}$ in air.

\section{Sorting and IVM of GV-stage oocytes}

GV oocytes were scored for the presence/absence of a Hoechst-positive ring of heterochromatin surrounding the nucleolus, leading to allocation in two classes (Surrounded Nucleolus, SN; Not Surrounded Nucleolus, NSN). Scoring was performed while holding the oocytes in Hepes-buffered CZB medium in the presence of $0.1 \mu \mathrm{g} / \mathrm{mL}$ Hoechst 33342 on the stage of an inverted microscope (Nikon TE 2000) fitted with an EXFO light source. After sorting, oocytes were allowed to progress to MII by culturing them in $\alpha$-MEM medium (Sigma cat. No. M4526) supplemented with $0.2 \%$ BSA (w/v) and $50 \mathrm{mg} / \mathrm{mL}$ gentamicin. Incubation took place at $37^{\circ} \mathrm{C}$ under $5 \% \mathrm{CO}_{2}$ and $5 \% \mathrm{O}_{2}$ (balance $\mathrm{N}_{2}$ ). This low-oxygen atmosphere was achieved in a homemade plastic chamber positioned on the shelf of the incubator and connected to a $5 / 5 / 90 \mathrm{CO}_{2} / \mathrm{O}_{2} / \mathrm{N}_{2}$ tank.

\section{Parthenogenesis}

Parthenogenetic activation of MII oocytes was performed for 6 hours in $\mathrm{Ca}$ free $\alpha$-MEM medium supplemented with $10 \mathrm{mM} \mathrm{SrCl} 2$. Cytochalasin B $(5 \mu \mathrm{g} / \mathrm{mL}$; Calbiochem cat. No. 250233) was also added to the medium so as to prevent the extrusion of a pseudo polar body and thereby obtain diploid parthenotes.

\section{Intracytoplasmic sperm injection}

The upper layer $(100 \mu \mathrm{L})$ of the sperm swim-up (30 min) suspension was collected and incubated as a drop under pre-equilibrated mineral oil for 2-3 $\mathrm{h}$ to allow for capacitation and acrosome reaction. Single sperm heads were injected into MII oocytes using a piezo drill (PrimeTech). ${ }^{11}$

\section{Embryo culture}

Parthenogenetic and ICSI-fertilized embryos were cultured 4 days in $500 \mu \mathrm{L}$
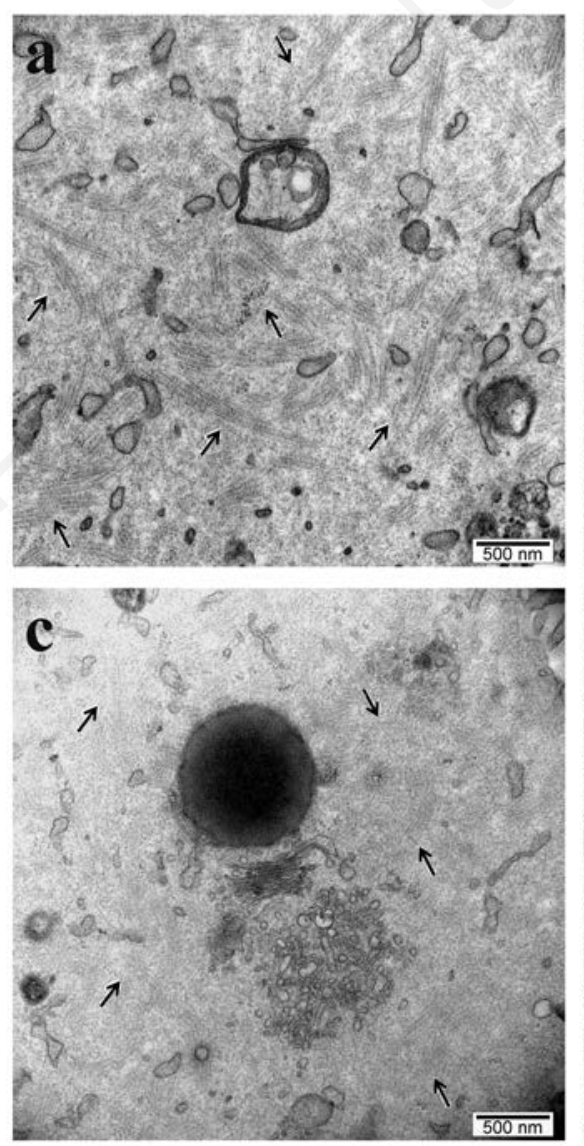

Figure 1. Representative TEM images of a) parthenogenetic NSN-derived 2-cell embryo and b) parthenogenetic SN-derived 2-cell embryo; c) NSN-derived 2-cell embryo obtained by ICSI; d) SN-derived 2-cell embryo obtained by ICSI. Arrows point to cytoplasmic lattices.
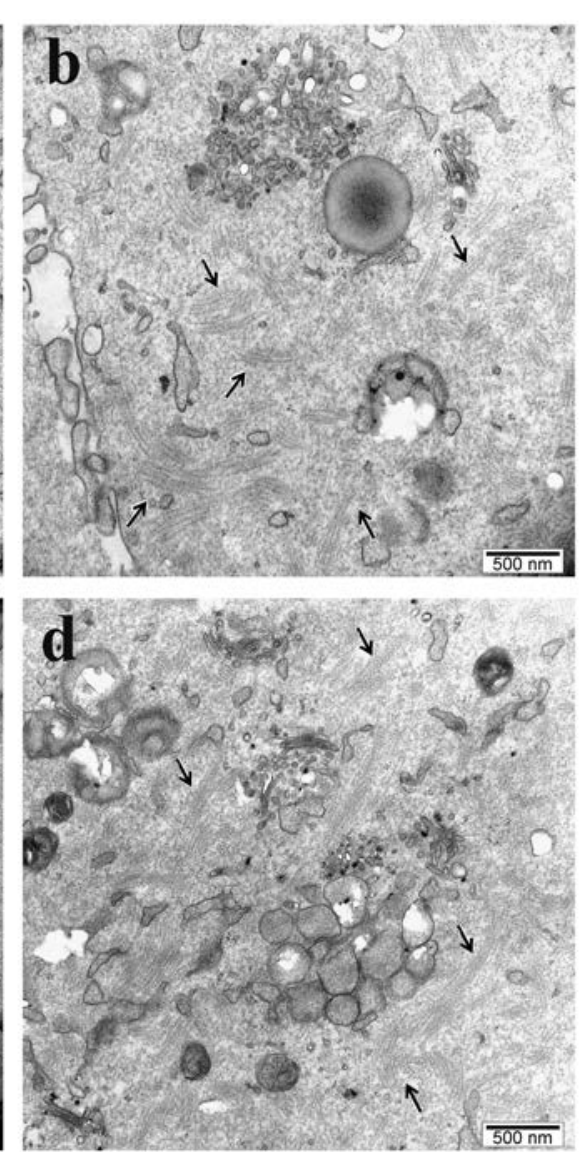

$\alpha$-MEM (supplemented with $0.2 \%$ BSA (w/v) and $50 \mathrm{mg} / \mathrm{mL}$ gentamicin) in 4-well plates (Nunc) without oil overlay, under $5 \%$ $\mathrm{CO}_{2}$ in air at $37^{\circ} \mathrm{C}$.

\section{Transmission electron microscopy}

At the 2-cell stage, a total of 82 embryos (58 SN, $24 \mathrm{NSN}$ ) and 48 embryos (30 SN, $18 \mathrm{NSN}$ ) were collected after parthenogenesis and ICSI, respectively. They were fixed in $2 \%$ paraformaldehyde, $2 \%$ glutaraldehyde in $0.1 \mathrm{M}$ cacodylate buffer, $\mathrm{pH} 7.2$ for three hours at room temperature. Next the samples were adsorbed to poly-L-lysin coated coverslips. ${ }^{12}$ Subsequently samples were post-fixed in $1 \%$ osmium tetroxide, $1.5 \%$ potassium ferrocyanide in $0.1 \mathrm{M}$ cacodylate buffer, $\mathrm{pH}$ 7.2. Then they were stepwise dehydrated in ethanol, including en bloc $0.5 \%$ uranyl acetate staining during $70 \%$ ethanol. The coverslips were flat embedded in epon and polymerized. From the hardened sample the cover slip was removed and the epon bloc was ultrathin-sectioned at $70 \mathrm{~nm}$. Sections were collected on copper grids and stained 
with lead. The samples were analyzed on a FEI-Tecnai 12 electron microscope (FEI, Eindhoven, The Netherlands) and imaged on a side-view camera (Veleta, EMSIS, Münster, Germany).

\section{Quantitative evaluation of CPLs}

Cytoplasmic lattices were quantitatively evaluated by Image J software (Rasband, W.S., ImageJ, U. S. National Institutes of Health, Bethesda, Maryland, USA, https://imagej.nih.gov/ij/, 1997-2016).

Two independent operators evaluated 10 randomly selected areas of the cytoplasm for each of the SN and NSN embryoderived types (Parthenotes and ICSI). The instrumental settings for the CPLs grey level evaluations was done independently by the two operators; the calculated integrated density was reported in arbitrary units normalized to the selected area (mean value \pm standard deviation). The two-tail test was used to analyze the distribution of the data that have a Gaussian distribution. Means and standard deviations were compared with the Student's $t$-test; differences were considered statistically significant when $\mathrm{P}<0.05$.

\section{Results}

Our main goal in this study is to contribute to detail the underlying cytological and molecular events controlling the very early embryo development, by taking a close examination of SN and NSN derived embryos on the ultrastructural level: we envision that the one and same biological machinery controls both the prospective embryo development and the arrest at the 2/4-cell stage.

Transmission electron microscopy analyses showed that CPLs are distributed throughout the cytoplasm of 2-cell embryos derived from both SN and NSN oocytes, without any appreciable difference between the two embryo production systems, as shown in Figure 1 a-d. The quantitative evaluations of the CPLs showed no significant differences $(\mathrm{P}>0.05)$ among any of the experimental conditions considered (Figure 2).

In addition to the CPLs we noticed one more feature that seems to differentiate between the $\mathrm{SN}$ and NSN-derived embryos, also in respect to apoptosis: the abundant presence of vesicular mitochondria known to be associated with the cytochrome c release during the initial phases of apoptosis. These organelles have been observed mainly in the cytoplasm of NSN-derived 2cell embryos, irrespective of the experimental conditions used (Figure 3). It is known that partial down-regulation of aerobic metabolism in both oocytes and embryos causes a reduced embryo viability pointing out the role exerted by mitochondria on the cytoplasmic regulation of preimplantation embryo development. ${ }^{13}$

\section{Discussion}

We previously reported that the cytoplasm of mouse and human SN and NSN $\mathrm{GV}$ oocytes is differentially enriched in CPLs, ${ }^{7,8}$ thus correlating this feature (low number) with the inability of NSN derived embryos to proceed beyond the 2/4-cell stage of development. The detailed ultra- structural analysis of SN and NSN 2-cell embryos obtained by parthenogenesis and ICSI is consistent with a model in which CPLs can form de novo during a specific timeline occurring during or immediately after the transition GV-zygote.

The important implication of our observation is that CPLs alone are not responsible for 2/4-cell embryos developmental arrest, contrary to previous assertions ("CPLs do not form after fertilization" and "the absence of the CPLs ultimately results in the development arrest" lacking CFP1 showed defects in CPLs formation that, among others, are responsible for defects in the acquisition of developmental competence after fertilization"). ${ }^{14}$ In

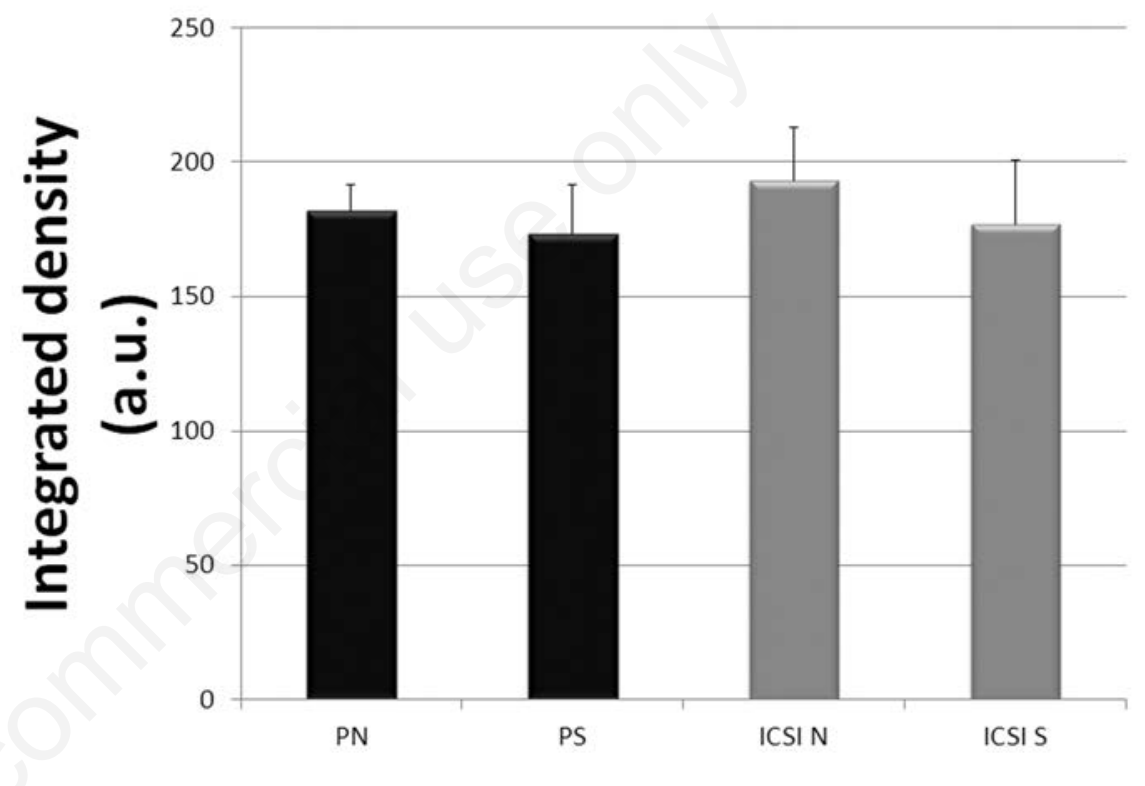

Figure 2. Integrated density evaluation (mean value \pm SD in arbitrary units, a.u.) of detectable CPLs in cytoplasmic representative areas (see materials and methods) of PN and PS (Parthenogenetic activated surrounded $(S)$ and not-surrounded $(N)$ nucleolus oocytes) and ICSI S and ICSI N (Intracytoplasmic Sperm Injected SN and NSN oocytes).
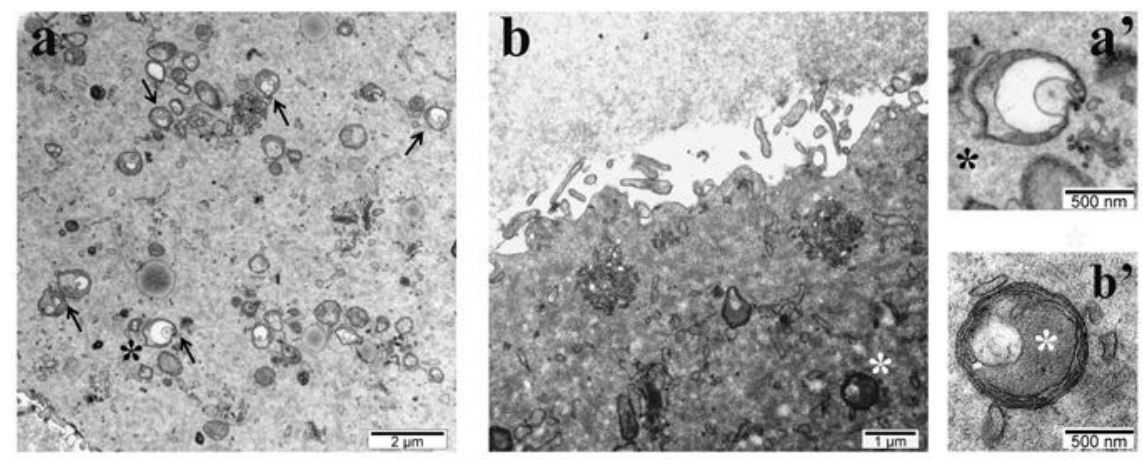

Figure 3. Representative TEM images of a) parthenogenetic NSN-derived 2-cell embryo with vesicular mitochondria (black arrows and black asterisk); b) $\mathrm{SN}$-derived 2-cell embryo obtained by ICSI showing non-defective mitochondria (white asterisk); a' and b' magnification of a vesicular and normal mitochondria respectively. 
order to link CPLs functions to the acquisition of oocyte genome architecture supportive of embryo development, we traced the development of embryos derived from SN and NSN GV oocytes, followed by two levels of inquiry. Firstly, we wished to confirm the well-known distinction whereby SN oocytes support development past the 2-cell stage, whereas NSN oocytes arrest at the 2cell stage. We employed ICSI so as not to lose any oocytes to a failure of fertilization, which is not uncommon among in vitromatured oocytes due to a hardening of the zona pellucida. Secondly, we examined the ultrastructure of these embryos, expecting to observe CPLs only in 2-cell embryos that were derived from SN oocytes. We employed chemical activation (resulting in diploid parthenogenesis) so as to preserve the pristine nature of the oocytes in respect to the CPLs (which are of maternal origin) and exclude any detrimental effects of the sperm injection on the ultrastructure. To our surprise, TEM analyses revealed the presence of CPLs in 2-cell embryos derived from both SN and NSN oocytes without any significant statistical difference (Figures 1 and 2).

We are aware that these preliminary observations require further investigations to fully understand this fascinating and challenging biological phenomenon. Nevertheless, we felt the urgent need to show that factors (i.e., CPLs), hitherto considered responsible for embryo arrest at the 2-cell stage, are actually not related to this outcome. Instead, we wish to shift the attention to other different organelles/mechanisms (i.e., abnormal mitochondria) that could, together with others, be the real player(s) of this intricate and extremely fascinating process that is the preimplantation embryo development.

These results prelude to further research with the ultimate aim to find new markers for better (non-invasive) oocyte selection, toward preferential therapeutic use of SN oocytes in assisted reproductive technology procedures.

\section{References}

1. Combelles C, Carabatsos MJ, Rajendra Kumar T, Matzuk MM, Albertini DF. Hormonal control of somatic cell oocyte interactions during ovarian follicle development. Mol Reprod Dev 2004; 69:347-55.

2. De La Fuente R. Chromatin modifications in the germinal vesicle $(\mathrm{GV})$ of mammalian oocytes. Dev Biol 2006; 292:1-12.

3. Kan R, Yurttas P, Kim B, Jin M, Wo L, Lee B, et al. Regulation of mouse oocyte microtubule and organelle dynamics by PADI6 and the cytoplasmic lattices. Dev Biol 2011;350:311-22.

4. Li L, Baibakov B, Dean J. A subcortical maternal complex essential for preimplantation mouse embryogenesis. Dev Cell 2008;15:416-25.

5. Esposito G, Vitale AM, Leijten FP, Strik AM, Koonen-Reemst AM, Yurttas P, et al. Peptidylarginine deiminase (PAD) 6 is essential for oocyte cytoskeletal sheet formation and female fertility. Mol Cell Endocrinol 2007;273:25-31.

6. Yurttas P, Vitale AM, Fitzhenry RJ, Cohen-Gould L, Wu W, Gossen JA, et al. Role for PADI6 and the cytoplasmic lattices in ribosomal storage in oocytes and translational control in the early mouse embryo. Development 2008; 135:2627-36.

7. Monti M, Zanoni M, Calligaro A, Ko MSH, Mauri P, Redi CA. Developmental arrest and mouse antral not-surrounded nucleolus oocytes. Biol Reprod 2013;88:1-7.

8. Monti M, Calligaro A, Behr B, Rejo Pera R, Redi CA, Wossidlo M. Functional topography of the fully grown human oocytes. Eur J Histochem 2017;61:2769.

9. Kim B, Kan R, Anguish L, Nelson LM, Coonrod SA. Potential role for MATER in cytoplasmic lattice formation in murine oocytes. Plos One 2010;5: e12587.

10. Liu YJ, Nakamura T, Nakano T. Essential role of Dppa3 for chromatin condensation in mouse oocytogenesis. Biol Reprod 2011;86:1:8.

11. Pfeiffer MJ, Balbach ST, Esteves TC, Crosetto N, Boiani M. Enhancing somatic nuclear reprogramming by Oct4 gain-of-function in cloned mouse. Int $\mathrm{J}$ Dev Biol 2011;54:1649-57.

12. Mildner K, Zeuschner D. Keep your feet on the ground! Tips \& tricks for keeping cells in position during sample preparation for EM. Imaging and microscopy 2017;38:40.

13. Thouas GA, Trounson AO, Wolvetang EJ, Jones GM. Mitochondrial Dysfunction in Mouse Oocytes Results in Preimplantation Embryo Arrest in Vitro. Biol Reprod 2004;71:1936-42.

14. Yu C, Fan X, Sha QQ, Wang HH, Li BT, Dai XX, et al. CFP1 regulates histone H3K4 trimethylation and developmental potential in mouse oocytes. Cell Reports 2017;20:1161-72. 